\title{
Feature-Based Face Detection: A Survey
}

\author{
Abbas M. Albakri ${ }^{1}$ \\ ${ }^{1}$ University of Information Technology \\ and Communications, Iraq \\ abbasmoh67@yahoo.com
}

\author{
Safaa O. Almamory ${ }^{2}$ \\ ${ }^{2}$ University of Information Technology \\ and Communications, Iraq \\ salmamory@uoitc.edu.iq
}

\author{
Hadeel H. Alfartosy ${ }^{3}$ \\ ${ }^{3}$ University of Information Technology \\ and Communications, Iraq \\ hhmmoe83@gmail.com
}

\begin{abstract}
Human and computer vision has a vital role in intelligent interaction with computer, face recognition is one of the subjects that have a wide area in researches, a big effort has been exerted in last decades for face recognition, face detection, face tracking, as yet new algorithms for building fully automated system are required, these algorithms should be robust and efficient. The first step of any face recognition system is face detection, the goal of face detection is the extraction of face region within image, taking into consideration lightning, orientation and pose variation, whenever this step accurate the result of face recognition will be better, this paper introduce a survey of techniques and methods of feature based face detection.
\end{abstract}

Keywords-Face recognition, Face detection, ASM, Viola-Jones.

\section{INTRODUCTION}

Face recognition system is a computerized software that uses images or frames of video to recognize individual faces within these images, it maybe verify or identify persons by matching input images with other stored images.[1][2][3].

A huge amount of papers that falls within face recognition subject and still increasing every day,1.100 papers is the result of google scholar in 2000 , while this number became 3.190 paper in 2007 [4], today the result is $3,190,000$, so it is clear that it is an important topic.

Biometrics are various body parameters like (iris, figure print, voice, face...etc.), many recognition techniques were developed using biometrics like iris recognition, finger print recognition, or even gate recognition (human walk behavior) and all these types of recognition falls within pattern recognition.[2][1]

Iris recognition is accurate but expensive for implementation, finger print is reliable but not suitable for non-collaborative individuals.[1], while the capturing of face image has no effort which makes it easiest and less expensive, also it does not require any physical interaction from the user[5]. But it remains to talk about accuracy and speed which requires going into more details within this paper but in general face recognition is low accuracy compared to the performance of finger print and iris recognition [3]

The first face recognition system was invented during 1964 and 1965, Woody Bledsoe, Helen Chan Wolf, and Charles Bisson worked on using computer to recognize faces of human, this project was named as man-machine, but there were a lot of difficulties in using that system like the size of database and dis capability of recognize faces in all conditions, inventor of this system used a standard frontal head derived from seven head measurements.

This work was continued by Standford research institute in 1966 precisely by Peter Hart, he performed experiments on 2000 images and showed that the project is really work.

In 1997, the project developed by Christoph and his graduate students from different universities, system funds is supported by United States army research laboratory, the software was sold with name ZN-Face and used by banks and airport, the system was good enough to identify person even with different face views and even see through mustaches, beards and glasses[6]

After that face recognition system widened its scope to take attention not only by programmers and engineers but also by neuroscientists because it has possible applications in computer vision communication and automatic access control system[7].

There are many papers that wrote in this topic but there is no paper that included face recognition stages and evaluating of this system under the same paper of face detection techniques, so the reader will gain a full idea in this field, also the measurement of results in researches that used in this paper were different and clattered, but we unified them to be clearer and we explained the reasons behind each result, that will give a reader a good knowledge for deciding which technique better for his work.

In the second section of this paper related work will be discussed then stages of recognition system will be explained in third section the stages of typical face recognition system will explored, fourth section will illustrate challenges of any recognition system, system test and evaluation is explained in fifth section, feature based face detection will explained in details in sixth section, then performance of face detection method will be summarized in table.

\section{RELATED WORK}

A lot of surveys and reviews that published in various world journals, some of these works are listed below to help readers finding them easily.

In 2002, M.-H. Yang et al. [8] wrote a survey on detecting face in images and classify single image detecting techniques into four catigouries (knowledge based,feature invariant, template matching, appearance based method), they gave a representive work for each approach, but because of the oldness of this papers thare are some new 
sub-approaches doesn't mentioned like Viola-Jones that we will explain in section VII.

Between (2003-2009) there are no paper written under this topic, but in 2010 "A Survey of Recent Advances in Face Detection" wrote by Cha Zhang et al.[9]but they focussed on Viola-Joines algorithm so it doesn't give the knowledge behind the tittle.

In 2014 "Studey and Analysis of Different Face Detection Techniques "[10] produced by M. Chauhan et al. this paper made studey of several existing face detection approaches and analyzed them. Each approach is compared with the other in terms of key evaluation.

Hiyam Hatem et al. [11] wrote a very good paper in this topic in 2015, in which they explain feature based face detection and gave a discription of 11 database used for face detecion analysis.

\section{STAGES OF RECOGNITION SYSTEM}

A typical face recognition system as in fig. 1 contains the following stage:

1. Image acquisition by camera: image can be acquired either from static photograph or frames from a sequence of video frames.

2. Preprocessing: remove noise from acquired image by applying some filters.

3. Face detection: there are thousand algorithm that applied for that purpose[12], in general face detection as we define before is to extract face area from original image.

4. Normalization: crop face as sub image from the whole image and make it suitable for the next process, which mean standardized it in terms of size, pose, illumination, landmarks of face, like eyes corners or size of nose, input image should be approximately the same image that saved in database, that will lead to make an accurate recognition process [13], just for normalization there are many research

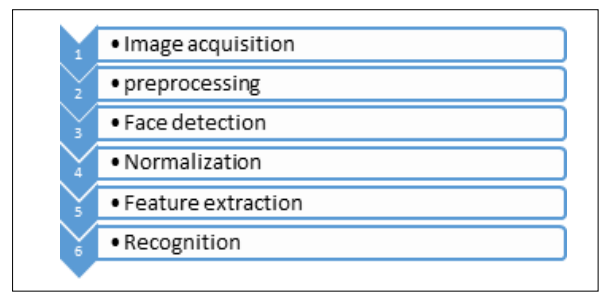

Fig. 1. Illustration of system stages

dealing with it.

5. Feature extraction: Extracting structural facial features like (eyes, nose, mouth... etc.)and the characteristics of each feature like nose size, skin, color of eye...etc. [1][14], in this phase features are stored as a mathematical representation and will then acts as the base for recognition task [13].

6. Recognition : Recognition or Matching phase is the last on the system by which give a decision for an input image either match or no match, there are many techniques that used for that purpose even artificial intelligent algorithms (like learning algorithm) are harnessed for making matching decisions [1][15][8].

\section{CHALLENGES OF THE SYSTEM}

Face detection depends on the proprieties of acquire image like noise and lightning condition, generally challenges can be listed as follow:

1. Pose: acquire image may be frontal face pose, 45 degree, upside, downside ...etc. and that surely will effect on the facial features [8][11] [1].

2. Facial expression: emotion of person primarily appears on his face (laughing, sadness, anger...etc.), all these motions significantly effect on facial features appearance, also the age of the person has a similar effect.

3. Illumination: different lightning environments is an important factor in detecting faces and that belong maybe to camera setting or different time image acquisition (night, day).

4. Occlusion: some objects can occlude some facial features like put one hand on mouth or warring scarf that hide a part of face...etc.

5. Temporary structural component: like presence or absence of beards, mustaches and glasses, all these considered as challenges in detecting faces because of variety of them in color, shape and size.

For all of these problems, no current face recognition system can handle all of these problems at the same time [3].

The aim of face detection is not only to find face in a proper image but also to localize face precisely within image and the location of each feature involved in that face like(eyes, nose, mouth...etc.)[8][12].

\section{V.SYSTEM TEST AND EVALUATION}

Typically face recognition system is used for two tasks: verification and identification. Verification or authentication is the easiest task, in which an individual image is matched with one saved image and to tell if it is the identity that claim to be. This is one-to-one matching process, the outcome of verification task is as follow:

1. Individual image matched: either as a false acceptance (in real it is not the same individual in database) or true acceptance (it is the same individual in data base.

2. Individual image not matched; either as false reject (in real it is the same individual in data base) or true reject (it is not the same individual in data base).

Identification task is more complex than verification task,

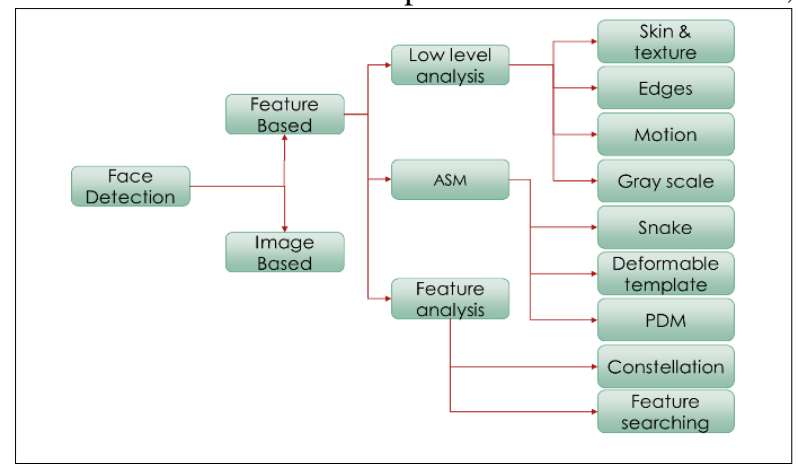

Fig. 2. Face detection techniques

identification is responsible for telling us who is this in the 
individual image and what is his identity. So it is one-tomany task. In addition there are difference between (I- close set identification in which the person should be identified, and (II- open set identification which is more complex because there is no certain if the person is exists in database or not, the outcome of identification task is as follow:

1. In close-set: there are three possibilities, either true positive (matched with correct person in database), false positive (matched with incorrect person in database) or false negative (not matched with any person in the database.

2. In open set: This time, there are four possibilities: either true positive (matched with correct person in database, false positive (matched with incorrect person in database, false negative (not matched with any person in database in spite of the matched image is in the database or true negative (not matched with any person in the database and there is no matched in database.

Depending on these possible results, two important metrics in recognizing system are considered to evaluate the system False Rejection Rate (FRR) and False Acceptance Rate (FAR). By these two metrics the system can be tested, whenever these rates are decreased the system will by better performance [1][2]

\section{FACE DETECTION TECHNIQUES}

It is a primarily first phase in face recognition system, its purpose is to find and localize face region from the background, and mostly the background is cluttered and merged with face color which makes it difficult to find face. Researchers classify face detection techniques according to different consideration, Authors [8][16][17] categorized method of face detection as a survey into four types. First is knowledge-based method which exploits human knowledge on typical face and encode this knowledge to generate roles in relationship between facial features. Second feature interval approach that exist whenever, pose, view point or lighting vary, third is template matching method which stores some face patterns and used in search of face weather image or facial feature separately. Fourth appearance based method. Which essentially rely on artificial intelligence learning algorithms (like neural networks, support vector machine ... etc.) this method find (model) or template from set of (training images) to be used after that for detect faces. An other manner in figure.2, for classify face detection technology into two types Image based approach and feature based approach [1][11], actually image based approach is the same of appearance based method and feature based method is the same invariant features method. But with different names.

Image based approach depends on artificial intelligence and statistical analysis (Neural network, linear subspace, PCA,SVM) [1][11], in this paper we will give a focus on feature based approach.

\section{FEATURE BASED APPROACHES}

This method is farther divided into three types.

1. low level analysis
Which analyze the basic image component like (intensity, color, texture, edge, motion...etc.) to detect face.

1.1. Skin Color and texture based analysis:

Color of human skin is good feature for detection, many systems exploit skin tones with choosing threshold carefully to detect face even in complex background. Author [18] proposed a method depending on skin color model, then apply Morphologic processing method and roughly filtering on extracted regions, the average detection time is $1.5132 \mathrm{~s}$, 424 successful detected image from 450 image.

Author[7]presents a project which segment image depending on skin color and classify each pixel in image into skin or non-skin, he obtained color skin value using 164 training faces in 7 images, his algorithm showed $93.3 \%$ of right detection rate, and $4.2 \%$ of false hit rate, and the average run time was 96 seconds.

Another proposed system [19]applied some types of noise removable then he formed a skin map, and search in each detected skin color region on two eye blobs, if eyes are founded then it is a face else it is regarded as a non-face, this method showed 2308 successful face detection from 2615 tested images which mean $88.26 \%$ true detection rate.

Just like skin color, skin also has a texture feature that can be exploit to isolate face from background,

In [20], a novel detection algorithim is proposed uses combination of edge and sckin color features this increase the effeciency of detecting faces and leading to decrease false acceptnece rate, this algorithm gain 21 false acceptness while it was 128 in case of using skin texture feature only.

\subsection{Edge based analysis}

Earliest face detection work used edge detection for facial feature extraction [1][8]by analyzing line drawing of the face and matched to face model to achieve correct detection , J. Wang [21] proposed a project that detect face using edge detection in images with simple background. He made image enhancement and filtering before applying a zerocrossing detector which is type of edge detection then made linking between detected edges, this system achieved $84.96 \%$ exact correct face detection.

\subsection{Motion based analysis}

Sometimes we need to detect faces in video in which we need to extract faces by detecting motion features [1][8], by frame difference the face could be detected regardless to background, Author [22] worked on real time video to verify liveness and to achieve lip reading of digits, also he used two different datasets to make experiment, $100 \%$ detection rate are gain for one dataset while the other one was $94.2 \%$ detection rate. Occasionally it is very good decision to exploit motion based feature for making face tracking furthermore face detection[23][24][25].

\subsection{Gray level based analysis}

Eyes, eyebrows, lips, nose tips and blobs of eyes, all these features are darker than skin color, so we can exploit that difference by gray level analysis to detect faces. Firstly image should converted into gray scale format, and may be obeyed to some types of filters then we should locate features using pixel intensity values[1][8][11]. 
Author [26] focused on locating facial features depending on gray level analysis, this project consist of three steps, first it extracts face depending on color of skin, then the image converted into YCbDr, third step is locating facial features using gray level intensity value, this system achieved $93 \%$ locating accuracy.

Automatic face location system using gray-scale images with complex backgrounds is proposed by D. Maio[27], the dataset contained 70 image, each one contain at least one face, just one image is missed detection with run time 0.078 second.

In [28], also a gray level feature used for detecting eyes and nose, the system is applied on four different datasets, successful nose locating rate was(91\%-99\%) and $(85 \%$ 98\%)rate was for eyes.

2. Active shape model(ASM)

It is point distributed model which form a face shape, used for detecting faces within images, these points represent face landmarks, and they are changeable in location with some tolerance, this type of face detection is further divided into three types:

2.1. Snakes

Locating boundary of head by snakes or active contour, also these contours can find other facial features boundaries, start point of snake should be initialized in a point of head boundary [11].

\subsection{Deformable Template}

This approach are developed to be more flexible than previous one, sometimes bad lightening cased problem in edge detection of head boundary in snake approach,

deformable template is rely on local valley, edges, brightness and peak, this template is flexible and can change its size to be able to detect face [1][11][8].

\subsection{Point distribution model (PDM)}

This approach creates a model which is compact parameterized descriptions of the shapes based on statistics, it is different from other ASMs because the contours of this model is discredited into a set of labeled points, the variations of these points parameterized to different shapes of face: size, pose.

Active shape model is proved to be a good tool for finding face landmarks and therefore a lot of systems exploit this approach to get a good accuracy in performing. L. H. Thai in [29] proposed a system that used ASM to locate face landmarks precisely with 68 points, he used Soble filter and canny edge detection for enhancing edges to make his work stronger and to find alignment of the face, using two datasets for his experiments, (14.021 and 11.751) was average error. ASM improved by[30], primarily for facial features extraction, because the normal ASM suffers from some limitations like poor model initialization, modeling the intensity of the local facial features, and alignment of the shape model, authors initialized the shape by finding the centers of features like nose and mouth, in the other hand they

used RGB color information to represent the local feature points, finally they applying $2 \mathrm{D}$ transformation in order to work for pose variation, this project is tested in two ways, first, made comparison between the error rates of standard ASM and a new one, second they applied face recognition using the extracted facial features from the two types of ASM and make a recognition process to measure the accuracy of each one, the average rate of minimum square error of standard ASM was $30 \%$ while the rate of enhanced ASM was $70 \%$, obviously there is good enhancement in new one.

\section{Feature analysis}

Furthermore divided into two types:

\subsection{Constellation analysis}

To decrease the difficulty of locating faces in various poses in cluttered background there is a group of features in facelike constellations statistical analysis which is strong analyzer in face detection. Author[31]proposed a system that uses statistical method for locating 15 features in face and apply his system on 150 images and gained 84\% performance rate.

\subsection{Features searching}

Also furthermore divided into two types:

\subsubsection{Viola-Jones}

It is the first method that proposed for real time object detection, it is invented by two students in the university of

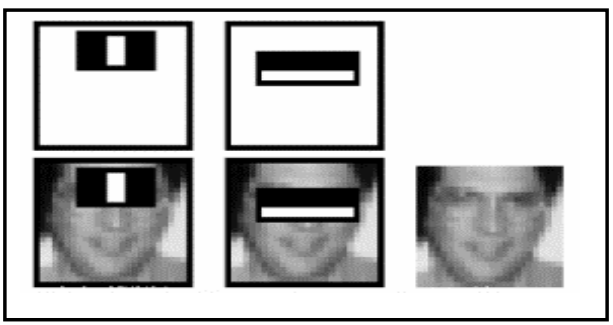

Fig. 3. Harr-like feature template

Cambridge in 2001[11][1], four steps are concluded in this method[81]:

First step: a Haar-like feature is a rectangle region in specific location in detection area of image called pattern, it sums up the pixel values in each region and computes the difference between these sums, Haar-like features are used to detect some face features as in figure.3, when image is scanned black region is replaced by +1 while white region is replaced by -1 , we start with any type and shift it to all image then increase the number of pixels, the input of this step is a $24 \times 24$ image and the output is a $\mathrm{d} \times 1$ scaler vector with its feature index ranging from 1 to $\mathrm{d}$.

Second step: integral image, which mean that the value at the pixel $(x, y)$ is the sum of pixels above and to the left of $(\mathrm{x}, \mathrm{y})$, as in fig.4, so the input of this step is $\mathrm{NxN}$ image and the output is another $\mathrm{NxN}$ image but after integral process.

\begin{tabular}{|l|l|l|}
\hline 1 & 1 & 1 \\
\hline 1 & 1 & 1 \\
\hline 1 & 1 & 1 \\
\hline
\end{tabular}$\longrightarrow$\begin{tabular}{|l|l|l|}
\hline 1 & 2 & 3 \\
\hline 2 & 4 & 6 \\
\hline 3 & 6 & 9 \\
\hline
\end{tabular}

Fig. 4. Integral image 
Third step: It is only few set of features will be useful among 160,000 features to identify a face (relevant features, irrelevant features), Adaboost is a machine learning algorithm which helps in finding only the best features among all 160,000 features. After these features are found a weighted combination of all these features is used in evaluating and deciding any given window has a face or not each of the selected features are considered okay to be include. The input of this step is an e $x$ e image (e>=24) with the parameters of the Harr-like features in the first step, the output is just feature value

Fourth step: Cascading, The basic principle of the violajones face detection algorithm is to scan the detector many times through the same image each time with a new size, that leads to scan non-face region repeatedly, the algorithm should constraint on discarding non faces quickly and spend more on time on probable face regions, so all the features are grouped into several stages where each stage has certain number of features, The job of each stage is used to determine whether a given sub window is definitely not a face or may be a face, a given sub window is immediately discarded as not face if it fails in any of the stages,fig.5.

In [32] viola-Joins algorithm is implemented and achieved $88.89 \%$ true acceptances and $11.11 \%$ false acceptance,

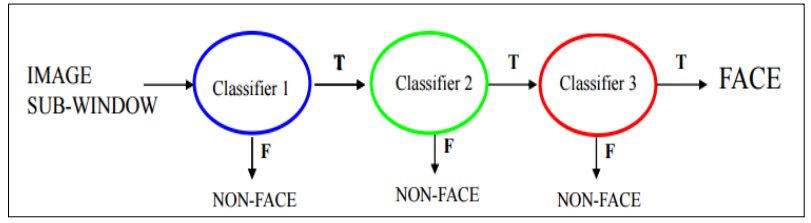

Fig. 5. Casscading

author [33]proposed a system for face recognition using Viola-Jones and correlation method, performance rate was $97.37 \%$.

\subsubsection{Gabor Filter}

A sinusoidal wave defines its impulse response multiplied by a Gaussian function. Because of the multiplicationconvolution property, the Fourier transform of a Gabor filter's impulse response is the convolution of the Fourier transform of the harmonic function and the Fourier transform of the Gaussian function. The filter has a real and an imaginary component representing orthogonal directions, the two components may be formed into a complex number or used individually.

In [34], 90\% performance rate is achieved using Gabor filter method, author [35] proposed system which commenced on convolving a face image with a series of Gabor filter coefficients at different scales and orientations, the result was $84.50 \%$ true acceptance.

\section{Performance of FACE DeTECTION METHODS}

Any system of detection or recognition is evaluated by some rates, these rates are: False Acceptance Rate (FAR) and
True Acceptance Rate (TAR), a list of works that surveyed in the paper summarized in the following table:

\section{TAble (1) PeRformant OF FACE DeTECTION METHOdS}

\begin{tabular}{|c|c|c|c|c|c|}
\hline \multirow[b]{2}{*}{ Author } & \multirow{2}{*}{\multicolumn{2}{|c|}{ Technique }} & \multicolumn{3}{|c|}{ Measurements } \\
\hline & & & FAR & TAR & $\begin{array}{l}\text { Run } \\
\text { Time } \\
\text { (s) }\end{array}$ \\
\hline $\begin{array}{l}\text { Inseong } \\
\text { Kim et } \\
\text { al.[36] }\end{array}$ & \multicolumn{2}{|c|}{$\begin{array}{l}\text { Skin Color and } \\
\text { texture }\end{array}$} & $4.2 \%$ & $93.3 \%$ & 96 \\
\hline $\begin{array}{l}\text { H. Lin et al. } \\
{[19]}\end{array}$ & \multicolumn{2}{|c|}{$\begin{array}{c}\text { Skin Color and } \\
\text { texture }\end{array}$} & $\begin{array}{c}12.67 \\
\%\end{array}$ & $88.26 \%$ & - \\
\hline $\begin{array}{c}\text { K. } \\
\text { Kollreider } \\
\text { et al. [21] }\end{array}$ & \multicolumn{2}{|c|}{ Edge based analysis } & $\begin{array}{c}3.47 \\
\%\end{array}$ & $84.96 \%$ & - \\
\hline \multirow{2}{*}{$\begin{array}{c}\text { K. } \\
\text { Kollreider } \\
\text { et al. [22] }\end{array}$} & \multirow{2}{*}{$\begin{array}{c}\text { Motion based } \\
\text { analysis }\end{array}$} & $\overrightarrow{0}$ & 0 & $100 \%$ & - \\
\hline & & $\tilde{\mathscr{0}}$ & $\begin{array}{c}1 \\
25 \times 1 \\
0-6\end{array}$ & $94.2 \%$ & - \\
\hline $\begin{array}{l}\text { D. Rana } \\
{[26]}\end{array}$ & \multicolumn{2}{|c|}{$\begin{array}{l}\text { Gray level based } \\
\text { analysis }\end{array}$} & - & $93 \%$ & - \\
\hline $\begin{array}{l}\text { D. Maio et } \\
\text { al. [27] }\end{array}$ & \multicolumn{2}{|c|}{$\begin{array}{c}\text { Gray level based } \\
\text { analysis }\end{array}$} & $\begin{array}{c}1.42 \\
\%\end{array}$ & $98.5 \%$ & 0.078 \\
\hline \multirow{4}{*}{$\begin{array}{l}\quad \text { M. } \\
\text { Hassaballah } \\
\text { et al. [28] }\end{array}$} & \multirow{4}{*}{$\begin{array}{l}\text { Gray level } \\
\text { based } \\
\text { analysis } \\
\text { (for eyes } \\
\text { detection) }\end{array}$} & $\bar{n}$ & - & $85.5 \%$ & \multirow{4}{*}{0.08} \\
\hline & & $\tilde{\tilde{\theta}}$ & - & $94.3 \%$ & \\
\hline & & $\tilde{\tilde{\theta}}$ & - & $98.4 \%$ & \\
\hline & & 苚 & - & $78 \%$ & \\
\hline \multirow{2}{*}{$\begin{array}{l}\text { L. H. Thai } \\
\text { [29] }\end{array}$} & \multirow{2}{*}{$\begin{array}{l}\text { Active } \\
\text { Shape } \\
\text { Model }\end{array}$} & $\vec{\circ}$ & $\begin{array}{c}10.54 \\
\%\end{array}$ & - & - \\
\hline & & $\tilde{\mathscr{0}}$ & $\begin{array}{c}7.17 \\
\%\end{array}$ & $\begin{array}{lll}- & - \\
\end{array}$ & - \\
\hline $\begin{array}{l}\text { M. Burl et } \\
\text { al. [31] }\end{array}$ & \multicolumn{2}{|c|}{$\begin{array}{c}\text { Constellation } \\
\text { analysis }\end{array}$} & - & $87 \%$ & - \\
\hline $\begin{array}{l}\text { F. Lobban } \\
\text { et al. [32] }\end{array}$ & \multicolumn{2}{|c|}{ Viola-Jones } & $\begin{array}{c}11.11 \\
\%\end{array}$ & $88.89 \%$ & - \\
\hline $\begin{array}{l}\text { S. Ranjeet } \\
\text { et al. [33] }\end{array}$ & \multicolumn{2}{|c|}{ Viola-Jones } & - & $97.37 \%$ & - \\
\hline $\begin{array}{c}\text { T. Barbu } \\
\text { [34] }\end{array}$ & \multicolumn{2}{|c|}{ Gabor Filter } & - & $90 \%$ & - \\
\hline $\begin{array}{l}\text { A. Bhuiyan } \\
\text { et al. [35] }\end{array}$ & \multicolumn{2}{|c|}{ Gabor Filter } & & $84.50 \%$ & $<1$ \\
\hline
\end{tabular}

\section{CONCLUSION}

This paper attempts to make a survey on feature based face detection and describe the details of each method, it is now clear that face detection and recognition is in the topic of world researches, there is still work to be done in spite of the great progress that has been made in last years, one day a robust face detection system will achieved passing all challenges. 


\section{REFERENCES}

[1] M. K. D. A. Datta, Face Detection and Recognition (Theory and Practice) - Eyal's Technical Blog, 1st ed. india: Taylor \& Francis Group, LLC, 2016.

[2] L. D. Introna and H. Nissenbaum, "Facial Recognition Technology. A Survey of Policy and Implementation Issues," Cent. Catastr. Prep. Response, New York Univ., vol. 74, no. 5, pp. 1-60, May 2009.

[3] A. W. Senior and R. M. Bolle, "Face Recognition and Its Application," Biometric Solut. Authentication An E-World, pp. 101-115, 2002

[4] M. J. Jones, "Face Recognition: Where We Are and Where To Go From Here," IEEJ Trans. Electron. Inf. Syst., vol. 129, no. 5, pp. 770-777, 2009.

[5] P. Kumar, M. Agarwal, and M. Nagar, "A Survey on Face Recognition System-A Challenge," Int. J. Adv. Res. Comput. Commun. Eng., vol. 2, no. 5, pp. 2167-2171, 2013.

[6] M. Ballantyne, R. S. Boyer, and L. Hines, "Woody BledsoeHis Life and Legacy," AI Mag., vol. 17, no. 1, pp. 7-20, 1996.

[7] S. Z. Li, "Face Detection," Learning, vol. 3, no. 9, pp. 1-6, 2005.

[8] M.-H. Yang, D. J. Kriegman, and N. Ahuja, "Detecting Faces In Image : A Survey," IEEE Trans. Pattern Anal. Mach. Intell., vol. 24, no. 1, pp. 34-58, 2002.

[9] C. Zhang and Z. Zhang, "A Survey of Recent Advances in Face Detection," 2010.

[10] M. Chauhan and M. Sakle, "Analysis of Different Face Detection Techniques," Int. J. Comput. Sci. Inf. Technol., vol. 5, no. 2, pp. 1615-618, 2014.

[11] H. Hatem, Z. Beiji, and R. Majeed, "A Survey of Feature Base Methods for Human Face Detection," Int. J. Control Autom., vol. 8, no. 5, pp. 61-78, 2015.

[12] R. Jafri and H. R. Arabnia, "A Survey of Face Recognition Techniques," J. Inf. Process. Syst., vol. 5, no. 2, pp. 41-68, 2009.

[13] A. Ramchandra and R. Kumar, "Overview Of Face Recognition System Challenges," Int. J. Sci. Technol. Res., vol. 2, no. 8, pp. 234-236, 2013.

[14] K. Yow and R. Cipolla, "Feature-based human face detection," Image Vis. Comput., 1997.

[15] W. Wójcik, K. Gromaszek, and M. Junisbekov, "Face Recognition: Issues, Methods and Alternative Applications."

[16] I. Khan and U. Mishra, "A Study of Techniques for Facial Detection and Expression Classification," Accent J. Econ. Ecol. Eng., vol. 1, no. 5, p. 17, 2016.

[17] H. Joshi and A. M. Bagade, "COMPARATIVE ANALYSIS OF FACE RECOGNITION TECHNIQUES,” pp. 64-71.

[18] D. Zhang, B. Wu, J. Sun, and Q. Liao, "A Face Detection Method Based on Skin Color Model," Proc. 11th Jt. Conf. Inf. Sci., pp. 1-5, 2008.

[19] H. Lin, S. Wang, S. Yen, and Y. Kao, "Face Detection Based on Skin Color and Neural Network Segmentation," IEEE, 2005.
[20] H. C. V. Lakshmi and S. P. Kulkarni, "Face Detection for Skintone Images Using Wavelet and Texture Features," vol. 3, no. 2, pp. 646-650, 2011.

[21] J. Wang and T. Tan, "A new face detection method based on shape information," Pattern Recognit. Lett., vol. 21, no. 6-7, pp. $463-471,2000$.

[22] K. Kollreider, H. Fronthaler, M. I. Faraj, and J. Bigun, "RealTime Face Detection and Motion Analysis With Application in 'Liveness ' Assessment," Analysis, vol. 2, no. 3, pp. 548-558, 2007.

[23] N. Ye and T. Sim, "Towards general motion-based face recognition," Proc. IEEE Comput. Soc. Conf. Comput. Vis. Pattern Recognit., pp. 2598-2605, 2010.

[24] N. Markuš, "Overview of algorithms for face detection and tracking," Fly.Srk.Fer.Hr.

[25] L. Yin, "Integrating active face tracking with model based coding," Pattern Recognit. Lett., vol. 20, no. 6, pp. 651-657, 1999.

[26] D. Rana, "Facial Feature Extraction of Color Image using Gray Scale Intensity Value," vol. 3, no. 3, pp. 1177-1180, 2014.

[27] D. Maio and D. Maltoni, "Real-time face location on grayscale static images," Pattern Recognit., vol. 33, no. 9, pp. 1525-1539, 2000.

[28] M. Hassaballah, K. Murakami, and S. Ido, "Eye and Nose Fields Detection From Gray Scale Facial Images," in MVA2011 IAPR, 2011, pp. 4-7.

[29] L. H. Thai, "Face Alignment Using Active Shape Model And Support Vector Machine,” Int. J. Biom., vol. 4, no. 6, pp. 224 234, 2012

[30] M. H. Mahoor, M. Abdel-Mottaleb, and A.-N. Ansari, "Improved Active Shape Model for Facial Feature Extraction in Color Images," J. Multimed., vol. 1, no. 4, pp. 21-28, 2006.

[31] M. Burl, T. Leung, and P. Perona, "Face localization via shape statistics," ... Work. Autom. Face ..., no. June, pp. 154 159,1995

[32] F. Lobban and S. Jones, "Implementing clinical guidelines (or not?)," Psychol. Psychother. Theory, Res. Pract., vol. 81, no. 4, pp. 329-330, Dec. 2008.

[33] S. Ranjeet and M. Kaur, "Face Recognition and Detection using Viola-Jones and Cross Correlation Method," vol. 4, no. 1, pp. 2498-2501, 2015.

[34] T. Barbu, "Gabor filter-based face recognition technique," Proc. Rom. Acad. Ser. A - Math. Phys. Tech. Sci. Inf. Sci., vol 11, no. 3, pp. 277-283, 2010.

[35] A. Bhuiyan and C. H. Liu, "On Face Recognition using Gabor Filters," Int. J. Comput. Electr. Autom. Control Inf. Eng., vol. 1, no. 4, pp. 51-56, 2007.

[36] and J. Y. I. K. Joon Hyung Shim, "Face Detection," in Handbook of Face Recognition, vol. 3, no. 9, New York: Springer-Verlag, 2005, pp. 13-37. 